\title{
Face Representation and Face Recognition using Optimized Local Ternary Patterns (OLTP)
}

\author{
G. Madasamy Raja ${ }^{\dagger}$ and V. Sadasivam*
}

\begin{abstract}
For many years, researchers in face description area have been representing and recognizing faces based on different methods that include subspace discriminant analysis, statistical learning and non-statistics based approach etc. But still automatic face recognition remains an interesting but challenging problem. This paper presents a novel and efficient face image representation method based on Optimized Local Ternary Pattern (OLTP) texture features. The face image is divided into several regions from which the OLTP texture feature distributions are extracted and concatenated into a feature vector that can act as face descriptor. The recognition is performed using nearest neighbor classification method with Chi-square distance as a similarity measure. Extensive experimental results on Yale B, ORL and AR face databases show that OLTP consistently performs much better than other well recognized texture models for face recognition.
\end{abstract}

Keywords: Face recognition, Local Binary Patterns (LBP), Local Ternary Patterns (LTP), Optimized Local Ternary Patterns (OLTP), Texture spectrum (TS)

\section{Introduction}

Automatic face recognition system plays a vital role in computer vision and pattern recognition applications. In the present world, biometric identification is unavoidable in various security applications such as surveillance, computerhuman interactions, video conference, airport security etc. As face is one of the most important biometrics, automatic face analysis is a necessary step in the field of security applications and research fields. Though many commercial application systems for face recognition are available in market, face recognition system is still an active and challenging topic because commercial applications will not deliver the correct result under uncontrolled environments like variations of pose, expression, illumination etc. As texture feature is an important factor in recognizing face images, this paper presents a new approach to face recognition, which is based on textural features.

For the last 25 years, many face representation approaches have been developed. Ekman and Friesen [1] used coding schemes for representing individual face representation whereas Turk and Pentland [2] used Eigen faces for face recognition. A new technique called Elastic Bunch Graph Matching (EBGM) approach was initiated by Wiskott et al. [3] in which a face can be represented as a graph by connecting many selected points in a face. A face authentication system based on Linear Discriminant

$\dagger$ Corresponding Author: Dept. of Information Technology, PET Engineering College, Vallioor, Tamil Nadu, India.

(anushpriya2004@yahoo.com)

* Dept. of Computer Science and Engineering, Manonmaniam Sundaranar University, Tirunelveli, Tamil Nadu, India.

(vsmsu@yahoo.com)

Received: July 31, 2015; Accepted: July 19, 2016
Analysis (LDA) was developed by Kittler et al. [4]. Hidden Markov Models (HMM) and Gaussian Mixture Models (GMM) were successfully used for face authentication by Cardinaux et al. [5]. The local intensity distributions are combined with the spatial information in the name of multi-resolution spatial histogram for face recognition and this idea was developed by Hadjidemetriou et al. [6].

Gabor filters were also successfully used for face identification by Yang et al. [7]. The Local Binary Pattern (LBP) operator, a well recognized texture feature has also been acting as a major role in face recognition application [8]-[10]. Texture features combined with geometrical features were proved to be successful in enhancing the quality of face recognition [11]. Likewise, Al-Osaimi et al. [12] combined texture feature with shape feature for face recognition.

Face recognition methods can be classified into features based methods and holistic methods [13]. Feature-based approaches identify and extract geometric relationships among the distinctive facial features such as the eyes, mouth, nose, etc., and then any pattern recognition techniques are employed to match faces using these measurements [14]. Holistic approaches identify faces using global representations, i.e., descriptions based on the entire image rather than on local features of the face. Linear Discriminant Analysis (LDA) and Principal Component Analysis (PCA) are some well known methods that are applied in the holistic approaches for face recognition. It was already proved that PCA can deliver better results than LDA especially when the size of the training data set is small in a comparative analysis done by Martinez and Kak [15]. Some recent successful techniques for face recognition $[16,17]$ which are based upon the 
Table 1. Overview of the important developments in the face recognition methods

\begin{tabular}{|c|c|c|}
\hline Methods & Description & Ref. No \\
\hline \multicolumn{3}{|c|}{ Holistic Approaches } \\
\hline Eigen faces & $\begin{array}{l}\text { Face recognition through Eigen } \\
\text { vectors }\end{array}$ & {$[2]$} \\
\hline LDA & $\begin{array}{l}\text { Face Authentication system based on } \\
\text { LDA }\end{array}$ & [4] \\
\hline $\begin{array}{c}\text { Multiresolution } \\
\text { Histogram }\end{array}$ & $\begin{array}{l}\text { Face recognition using the } \\
\text { histograms from multi resolution }\end{array}$ & {$[6]$} \\
\hline PCA & Face recognition through PCA & {$[15]$} \\
\hline \multicolumn{3}{|c|}{ Feature based Approaches } \\
\hline EBGM & $\begin{array}{l}\text { Face recognition by applying Graph } \\
\text { theory }\end{array}$ & {$[3]$} \\
\hline HMM and GMM & $\begin{array}{l}\text { Face recognition through Model- } \\
\text { based methods }\end{array}$ & {$[5]$} \\
\hline Multiscale Block LBP & $\begin{array}{c}\text { Face Recognition using Texture } \\
\text { model }\end{array}$ & [9] \\
\hline $\begin{array}{l}\text { Combined approach } \\
\text { of LBP and } \\
\text { geometrical features }\end{array}$ & $\begin{array}{l}\text { Face recognition through the } \\
\text { combined approach of texture with } \\
\text { geometry features }\end{array}$ & {$[11]$} \\
\hline $\begin{array}{l}\text { Fusion of shape and } \\
\text { texture features }\end{array}$ & $\begin{array}{l}\text { Face recognition through the } \\
\text { combined approach of texture with } \\
\text { shape features }\end{array}$ & {$[12]$} \\
\hline $\begin{array}{l}\text { Fusion of EBGM } \\
\text { with HOG }\end{array}$ & $\begin{array}{l}\text { Face recognition through the } \\
\text { combined approach of graphical } \\
\text { methods with HOG }\end{array}$ & {$[17]$} \\
\hline
\end{tabular}

EBGM gives the impression that EBGM is still acting as a widely accepted and well known feature-based approach for face recognition. Table 1 shows an overview of some major developments in the face recognition techniques from the literature.

The remainder of this paper is organized as follows: Section 2 describes the proposed face recognition algorithm and section 3 explains the various texture models used in this study. In section 4, experimental results are discussed and section 5 presents the conclusion remarks with the future scope of this study.

\section{Face Description Algorithm}

Generally all the face recognition system consists of two major phases namely training phase and testing phase. The following block diagram in Fig. 1 illustrates the processing chain in the face recognition algorithm used for this study.

\subsection{Training phase}

1. The input training image is selected.

2. The selected training image is divided into many non over-lapping regions.

3. The selected texture model is applied over the sub regions.

4. A one dimensional histogram is generated for each sub regions.

5. A pattern spectrum is formed by concatenating all the one dimensional histograms derived for all the sub regions. This pattern spectrum is the global face

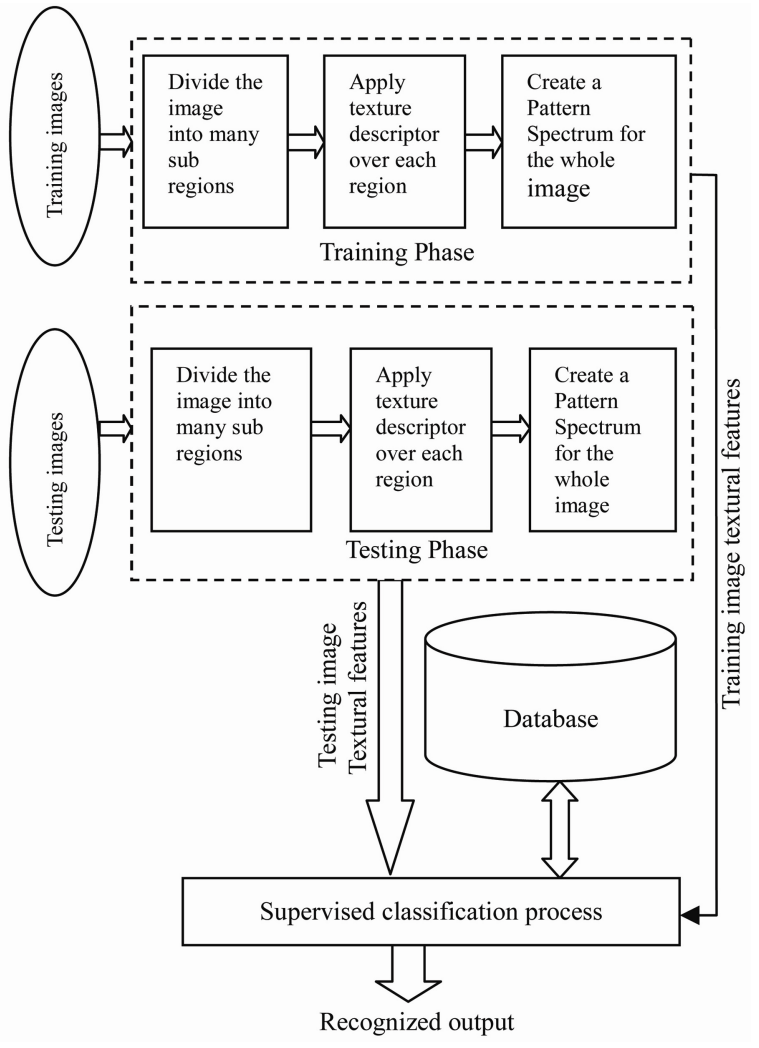

Fig. 1. The Block diagram of face recognition process

description of the given input training image.

6. This pattern spectrum is stored as the training feature in the database.

7. Steps 1 to 6 are performed repeatedly for all the training images and all the resulting features are stored in the database.

\subsection{Testing phase}

1. The input testing image is selected.

2. Steps 1 to 6 of training phase are performed to extract the feature of this testing image.

3. The resultant pattern spectrum will represent the face description of the given input testing image. This feature is compared with all the training features in the database by using a similarity measurement called Chi-square distance (2).

$$
\chi^{2}(x, y)=\sum_{i} \frac{\left(x_{i}-y_{i}\right)^{2}}{x_{i}+y_{i}}
$$

where $x$ is the pattern spectrum of the testing image sample and $y$ is the pattern spectrum of the training image sample, $x_{\mathrm{i}}$ is the frequency at bin i of testing image pattern spectrum and $y_{\mathrm{i}}$ is the frequency at bin $\mathrm{i}$ of training image pattern spectrum.

4. Choose the correct match from the trained pattern spectrum database for which the given testing pattern 
spectrum is having a value of minimum Chi-square distance.

\section{Texture Measures Used in This Study}

\subsection{Optimized Local Ternary Patterns (OLTP)}

Optimized Local Ternary Patterns (OLTP), a recent texture feature method which was already successfully applied for texture classification [18] and segmentation [19] is applied for face recognition in this paper. In OLTP, a texture image can be decomposed into a set of small units called patterns. As the texture model OLTP uses only optimal patterns (selected number of patterns), the length of the pattern histogram is maintained as optimum compared to other texture models. In OLTP, a pattern is represented by eight elements, each of which has one of three possible values $(0,1,5)$ that are obtained from a neighborhood of $3 \times 3$ pixels. Let $i_{c}, i_{1}, i_{2}, \ldots, i_{8}$ be the pixel values of a local $3 \times 3$ neighborhood region, where $i_{c}$ is the value of the central pixel and $i_{1}, i_{2}, \ldots, i_{8}$ are the pixel values of its 8 neighbors.

The following equation (2) defines the process for converting a local $3 \times 3$ neighborhood into its pattern representation $(\mathrm{P})$,

$$
P\left(i_{c}, i_{n}\right)=\left\{\begin{array}{lll}
0 & \text { if } & i_{n}<(1-\sigma) i_{c} \\
1 & \text { if } & (1-\sigma) i_{c} \leq i_{n} \leq(1+\sigma) i_{c} \\
5 & \text { if } & i_{n}>(1+\sigma) i_{c}
\end{array}\right.
$$

where $\sigma$ is a small scaling factor and it is assigned as 0.05 . Fig. 2 shows an example of transforming a sample $3 \times 3$ local neighborhood into a pattern unit. For a $3 \times 3$ local neighborhood region, the total number of different pattern strings will be $6561\left(3^{8}\right)$. So, by using this OLTP texture model a complete texture image can be described by a pattern histogram of 6561 bins that represents the occurrence frequency of pattern strings over the texture image.

Further, after many reliable experiments, it is observed that for a $3 \times 3$ local neighborhood of a texture image, only few pattern strings among these 6561 different pattern strings are frequently occurring compared to other pattern strings. So it is meaningless to allot separate bins for all the pattern strings because it may end in wastage of memory and wastage of time. Further, the analysis of these

\begin{tabular}{|l|l|l|}
\hline 252 & 172 & 168 \\
\hline 156 & 256 & 108 \\
\hline 244 & 380 & 332 \\
\hline
\end{tabular}

(a)

\begin{tabular}{|l|l|l|}
\hline 1 & 0 & 0 \\
\hline 0 & $\bigotimes$ & 0 \\
\hline 1 & 5 & 5 \\
\hline
\end{tabular}

(b)

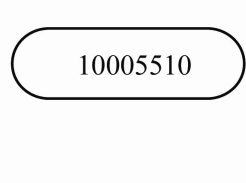

(c)
Fig. 2. Example of transforming a $3 \times 3$ neighborhood to a pattern string by using OLTP texture model: (a) $3 \times 3$ Local Region; (b) Pattern Matrix; (c) Pattern String frequently occurring pattern strings found that all these pattern strings have uniform circular structure and they are rotation invariant. To identify these frequently occurring pattern strings which are rotation invariant, consider a uniformity measure ' $U$ ' which corresponds to the number of spatial transitions circularly among the sub patterns in the pattern strings. The uniformity measure ' $U$ ' is defined as

$$
\begin{aligned}
& \mathrm{U}=\mathrm{F}\left(\mathrm{P}\left(\mathrm{i}_{\mathrm{c}}, \mathrm{i}_{1}\right), \mathrm{P}\left(\mathrm{i}_{\mathrm{c}}, \mathrm{i}_{8}\right)\right)+\sum_{\mathrm{n}=2}^{8} \mathrm{~F}\left(\mathrm{P}\left(\mathrm{i}_{\mathrm{c}}, \mathrm{i}_{\mathrm{n}}\right), \mathrm{P}\left(\mathrm{i}_{\mathrm{c}}, \mathrm{i}_{\mathrm{n}-1}\right)\right) \\
& \text { where } \mathrm{F}(\mathrm{A}, \mathrm{B})= \begin{cases}1 & \text { if }|A-B|>0 \\
0 & \text { otherwise }\end{cases}
\end{aligned}
$$

For example, the pattern string 11111111 has U value of 0 , the pattern string 00000055 has $U$ value of 2 and the pattern string 55501111 has $U$ value of 3. Patterns with $U$ value of less than or equal to 3 are considered as "Uniform Pattern Strings" and totally 45 uniform pattern strings are available. It should be noted that the elements of the uniform pattern strings are either 0 or 1 or 5 or any combinations of these values. The implementation of a new idea to calculate the Transition Length $(\rho)$ among the uniform patterns and the introduction of a new concept called Level of Optimality $\left(\mathrm{L}_{\mathrm{opt}}\right)$ have paved the way to select the subset of uniform patterns which are designated as optimal patterns.

If the number of occurrences (cardinality) of 0,1 and 5 are represented by $\operatorname{Card}(0), \operatorname{Card}(1)$ and $\operatorname{Card}(5)$ respectively, then the level of optimality for a particular uniform pattern string can be computed as,

$$
L_{\text {opt }}=\frac{1+\min \{\operatorname{Card}(0), \operatorname{Card}(1), \operatorname{Card}(5)\}}{\max (\rho)}
$$

where $\rho$ refers to the transition length among the subpatterns in the uniform pattern strings. Table 2 contains the transition lengths of various sub-patterns that are available in the uniform pattern strings.

Optimized Local Ternary Patterns (OLTP) texture model

Table 2. Details of the transition length $(\rho)$ for the subpatterns of uniform pattern strings

\begin{tabular}{c|c|c}
\hline \multicolumn{3}{|c}{ Transition Length } \\
\hline S.No & Sub-pattern type & Transition Length $(\rho)$ \\
\hline 1 & $' 01 '$ & 1 \\
\hline 2 & $' 10 '$ & 1 \\
\hline 3 & $' 15 '$ & 1 \\
\hline 4 & $' 51 '$ & 1 \\
\hline 5 & $' 05 '$ & 2 \\
\hline 6 & $' 50 '$ & 2 \\
\hline 7 & $' 015 '$ & 1 \\
\hline 8 & $' 510 '$ & 2 \\
\hline 9 & $' 105 '$ & 2 \\
\hline 10 & $' 150 '$ & 2 \\
\hline 11 & $' 051 '$ & 2 \\
\hline 12 & $' 501 '$ & \\
\hline
\end{tabular}


that uses only optimal set of uniform patterns to represent a local image texture is defined as:

$$
\text { OLTP }= \begin{cases}\sum_{\mathrm{i}=1}^{8} \mathrm{P}\left(\mathrm{i}_{\mathrm{c}}, \mathrm{i}_{\mathrm{n}}\right) & \text { if } \mathrm{U} \leq 3 \text { and } \mathrm{L}_{\mathrm{opt}} \geq 2 \\ 25 & \text { otherwise }\end{cases}
$$

This recently proposed OLTP uses only 24 unique optimal patterns for texture representation and it groups all other patterns under one label 25 which are termed as "suboptimal" patterns. Therefore the dimension of pattern spectrum has been reduced from 6561 to 25 optimal set of patterns. The occurrence frequency of 25 optimal patterns of a texture image is "OLTP Pattern Spectrum" or "OLTP Pattern Histogram", which reflects the texture information of the corresponding image.

\subsection{Texture Spectrum (TS)}

The Texture Spectrum method introduced by $\mathrm{He}$ and Wang [20], is based on the computation of the relative intensity relations between the pixels in a small neighborhood. The texture spectrum method which characterizes the original image with the help of image's texture characteristics will be the result after the application of the texture spectrum methodology to a given digital image. Texture spectrum method uses a basic concept called Texture Unit (TU). A texture unit is characterized by eight pixels each of which has one of three possible values $(0,1,2)$ obtained from a neighborhood of $3 \times 3$ pixels. If the intensity value of the central pixel is considered as $\mathrm{X}_{0}$ and the intensity value of each neighboring pixel is considered as $X_{i}$, the corresponding texture unit for the set that is considered as the smallest complete unit of the image under consideration is:

$\mathrm{TU}=\left\{\mathrm{E}_{1}, \mathrm{E}_{2}, \mathrm{E}_{3}, \ldots, \mathrm{E}_{8}\right\}$ where

$$
E_{i}= \begin{cases}0 & \text { if } X_{\mathrm{i}}<X_{0} \\ 1 & \text { if } X_{\mathrm{i}}=X_{0} \text { fori }=1,2, \ldots 8 \\ 2 & \text { if } X_{\mathrm{i}}>X_{0}\end{cases}
$$

$E_{i}=$ The $i_{\text {th }}$ element of texture unit set $T U=\left\{E_{1}, E_{2}, \ldots E_{8}\right\}$.

From the above formula, each element can be assigned one of three possible values so that the total number of possible texture units for the eight elements can be anticipated as $3^{8}=6561$. In the texture spectrum method, texture units are numbered by the following equation

$$
N_{T U}=\sum_{i=1}^{8} E_{i} 3^{i-1}
$$

where $\mathrm{N}_{\mathrm{TU}}$ is the texture unit number. Fig. 3 gives an example of transforming a neighborhood to a texture unit

\begin{tabular}{|l|l|l|}
\hline $\mathrm{X}_{1}$ & $\mathrm{X}_{2}$ & $\mathrm{X}_{3}$ \\
\hline $\mathrm{X}_{8}$ & $\mathrm{X}_{0}$ & $\mathrm{X}_{4}$ \\
\hline $\mathrm{X}_{7}$ & $\mathrm{X}_{6}$ & $\mathrm{X}_{5}$ \\
\hline
\end{tabular}

(a)

\begin{tabular}{|l|l|l|}
\hline 90 & 56 & 74 \\
\hline 56 & 70 & 62 \\
\hline 98 & 70 & 54 \\
\hline
\end{tabular}$\Rightarrow$\begin{tabular}{|c|c|c|}
\hline 2 & 0 & 2 \\
\hline 0 & - & 0 \\
\hline 2 & 1 & 0 \\
\hline
\end{tabular}

(b)
Fig. 3. Example for texture unit transformation: (a) Ordering of $3 \times 3$ neighbourhood; (b) Transformation of example $3 \times 3$ neighbourhood into a texture unit

\begin{tabular}{|l|l|l|}
\hline $\mathrm{X}_{1}$ & $\mathrm{X}_{2}$ & $\mathrm{X}_{3}$ \\
\hline $\mathrm{X}_{8}$ & $\mathrm{X}_{0}$ & $\mathrm{X}_{4}$ \\
\hline $\mathrm{X}_{7}$ & $\mathrm{X}_{6}$ & $\mathrm{X}_{5}$ \\
\hline
\end{tabular}$\quad$\begin{tabular}{|l|l|l|}
90 & 56 & 74 \\
\hline 56 & 70 & 62 \\
\hline 98 & 70 & 54 \\
\hline
\end{tabular}$\Rightarrow$\begin{tabular}{|l|l|l|l|}
\hline 1 & 0 & 1 \\
\hline 0 & & 0 \\
\hline 1 & 1 & 0 \\
\hline
\end{tabular}

(a)

Fig. 4. An example of LBP computation: (a) Ordering of $3 \times 3$ neighborhood; (b) Transformation of example $3 \times 3$ neighborhood into a LBP pattern

with the texture unit number as 1721. The occurrence distribution of the all the texture units is called Texture Spectrum (TS) which can be used for characterizing the texture available in a digital image.

\subsection{Local Binary Patterns (LBP)}

As the Texture spectrum method uses very large number of patterns, another texture model namely Local Binary Patterns (LBP) operator was introduced by Ojala et al. [21] which was based on a $3 \times 3$ neighborhood with less number of patterns.

LBP operated with eight neighboring pixels using the center as a threshold. If a neighbor pixel value is less than the threshold then 0 is assigned to that particular neighbouring pixel otherwise it is assigned as 1 . Result of this operation is a binary number which can be formed starting from any position in the $3 \times 3$ region neighborhood. The histogram of this eight bit binary number contains information of the distribution of the edges, spots, and other local features in an image. This binary number is converted into a decimal value and this decimal value is assigned as the label for the pixel values. So, for a $3 \times 3$ neighborhood, a total of $256\left(2^{8}\right)$ different binary pattern strings can be generated and the entire image can be represented by a pattern histogram of 256 elements, which can be used as the texture descriptor. This process is shown in Fig. 4.

Even in the year 2015 [22], LBP acts as a leading role in face recognition and this proves that LBP is still a latest technique in the face recognition algorithms. So this paper considers Local Binary Patterns (LBP) as one of the texture models for the comparative analysis in finding the accuracy of the proposed face recognition system.

\subsection{Local Ternary Patterns (LTP)}

As LBP is sensitive to noise, a 3-valued pattern instead 
of a binary pattern namely Local Ternary Patterns (LTP) was introduced by Tan and Triggs [23]. When a 3x3 neighborhood around a centre pixel in an image is considered, the LTP operator takes the following form

$$
\begin{aligned}
& L T P=\sum_{\mathrm{n}=1}^{8} 3^{\mathrm{n}} s\left(i_{n}-i_{c}\right), \\
& s(u)=\left\{\begin{array}{lll}
-1 & \text { if } \quad u \leq i_{c}-t, \\
0 & \text { if } \quad i_{c}-t<u<i_{c}+t, \\
1 & \text { if } \quad u \geq i_{c}+t,
\end{array}\right.
\end{aligned}
$$

where $\mathrm{t}$ is a user-defined threshold, $\mathrm{n}$ is the number of neighboring pixels surrounding the center pixel $\mathrm{c}, \mathrm{i}_{\mathrm{c}}$ is the pixel value of $c$ and $i_{n}$ are the pixel values of $n$. In LTP, the difference between the centre pixel and neighboring pixel is encoded by three values $(1,0,-1)$ according to a predefined and fixed threshold $t$, and the length of the pattern histogram is very high $\left(3^{8}\right)$. To reduce the dimension of the pattern histogram, the ternary pattern is converted into binary pattern, by splitting it into its positive and negative parts. Two separate histograms for both positive and negative components are calculated and then the results are concatenated.

\section{Experimental Analysis}

The experiments were conducted on Yale B, ORL and AR face databases which contain face images under different lighting conditions with different poses which are very suitable for testing any face recognition system. Using nearest neighbor classifier method the high similarity match of the testing sample with any one of the training sample is identified and such match is validated as either correct or incorrect based on the supervised knowledge.

\subsection{Results on YALE B face database}

First the experiments are conducted on Yale B face database that consists of 38 subjects with 9 different poses and 64 different illuminations for every pose. This study uses only frontal face images and 15 subjects are taken for research. Four frontal images with controlled illumination variation from each selected subject are randomly selected for training and 16 frontal images from the remaining images of the same selected subjects are used for testing. All the selected images for face recognition are down sampled into $64 \times 64$ size and are converted into gray scale images before experiments. Fig. 5 shows some sample images which are selected for this experiment. This experiment compares the face recognition performance of four texture descriptors TS, LBP, LTP and OLTP in the form of Recognition Rate, which is shown in Table 3. From the above results on face recognition, it is noted that LBP
Table 3. Face Recognition accuracy results on Yale B Face Database

\begin{tabular}{c|c|c|c}
\hline $\begin{array}{c}\text { No.of Training } \\
\text { Images }\end{array}$ & $\begin{array}{c}\text { No.of Testing } \\
\text { Images }\end{array}$ & $\begin{array}{c}\text { Texture } \\
\text { Model }\end{array}$ & $\begin{array}{c}\text { Recognition } \\
\text { Rate(\%) }\end{array}$ \\
\hline \multirow{3}{*}{60} & \multirow{3}{*}{240} & TS & 56.54 \\
\cline { 3 - 4 } & & LBP & 78.33 \\
\cline { 3 - 4 } & & LTP & 80.42 \\
\cline { 3 - 4 } & & OLTP & 90.01 \\
\hline
\end{tabular}

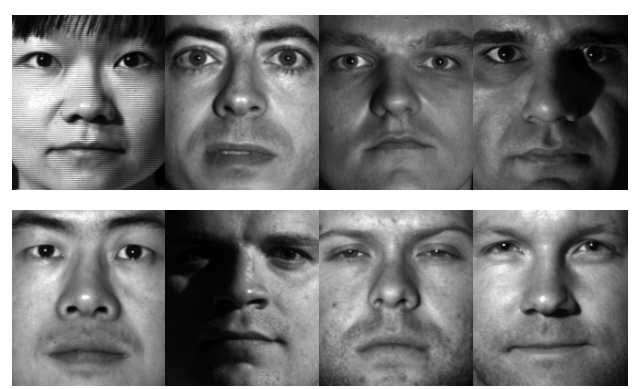

Fig. 5. Sample images-From Yale B face database

performs better than TS where as LTP performs superior to LBP. OLTP performs best over all the other texture methods namely, TS, LBP \& LTP. Generally a face recognition system is prone to two kinds of errors, namely False Acceptance Rate (FAR) and False Rejection Rate (FRR). FAR is a measurement tool to calculate how many impostors are falsely recognized as authentic users whereas FRR is a measurement tool that tells how many genuine users are falsely recognized as imposters by the face recognition system.

For the evaluation of these error rates (FAR \& FRR) of the newly proposed OLTP based face recognition algorithm, 15 selected subjects with 20 frontal face images from each subject of this Yale B face database is divided into two groups such as 9 known subjects and 6 unknown subjects. The system is trained with 45 images of known subjects that are 5 face images from each subject. Testing is done with balance 255 images of both known and unknown subjects. False Acceptance Rate (FAR) and False Rejection Rate (FRR) are calculated as follows

$$
\begin{aligned}
& \text { FAR }(\%)=100-\text { Recognition Rate } \\
& \text { FRR }(\%)=100-\text { Rejection Rate }
\end{aligned}
$$

where

$$
\begin{aligned}
& \text { Recognition Rate }(\%)=\frac{\text { No.of correct matches }}{\text { No. of test images }} \times 100 \\
& \text { Rejection Rate }(\%)=\frac{\text { No.of correct rejection }}{\text { No. of test images }} \times 100
\end{aligned}
$$

Fig. 6 shows the FAR and FRR produced by OLTP based face recognition method, for the various thresholds of similarity measurements for the Yale B face database. From these two error rates (FAR \& FRR), another important 
Table 4. Comparative analysis of EER values for Face Recognition

\begin{tabular}{c|c|c}
\hline S. No & Texture Model & Equal Error Rate (EER) $\%$ \\
\hline 1 & TS & 8.1 \\
\hline 2 & LBP & 6.2 \\
\hline 3 & LTP & 5.3 \\
\hline 4 & OLTP (proposed) & 4.0 \\
\hline
\end{tabular}

OLTP - Face Verification Performance

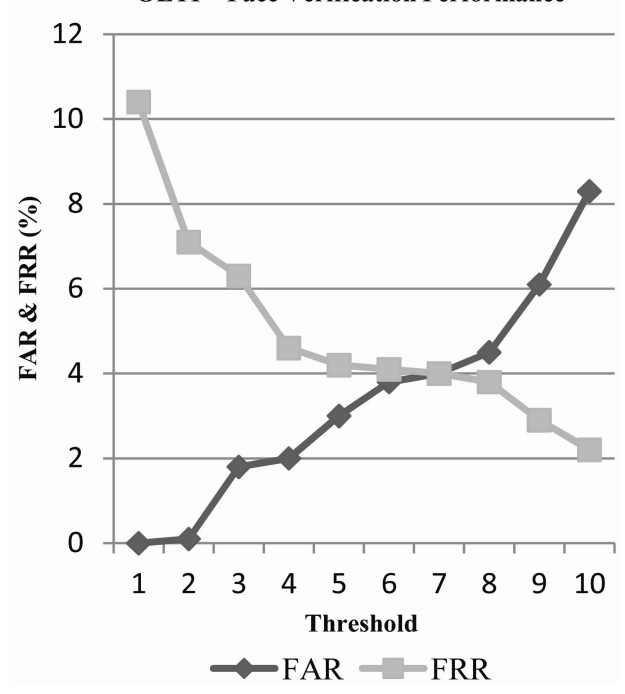

Fig. 6. False Acceptance Rate (FAR) and False Rejection Rate (FRR) for various thresholds of similarity measurements when OLTP texture model was used in the face recognition system on Yale $B$ face database

technical value called EER (Equal Error Rate) can be derived. EER is the intersection point at which FAR and FRR are equal. It is already understood that, the lower the EER value is, the better is the verification or recognition accuracy. Table 4 summarizes the EER values achieved by the various texture models for Yale B database. So, from Table 4, it can be concluded that among all the texture models considered for this experiment (TS, LBP, LTP and OLTP), EER value for the new texture model OLTP is the lowest (4\%), so the texture model OLTP can be used as a good face recognition tool also in addition to texture analysis. Genuine Acceptance Rate (GAR) is another important measurement for finding the accuracy of any biometric system. GAR is calculated as follows:

\section{Genuine Acceptance Rate = 1- False Rejection Rate}

To prove the efficiency of OLTP on face recognition system in a comprehensive way, this research finds the GAR values for the varying FAR values for all the texture models that are used in this comparative study. For this evaluation study, 15 selected subjects with 20 frontal face images from each subject of this Yale B face database is again divided into two groups such as 8 known subjects

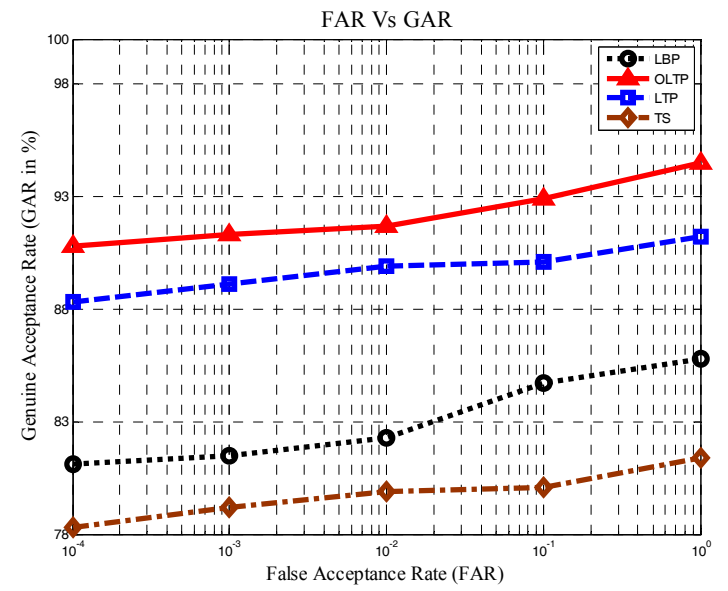

Fig. 7. ROC curve for the matching of FAR and GAR for various texture models on Yale B face database

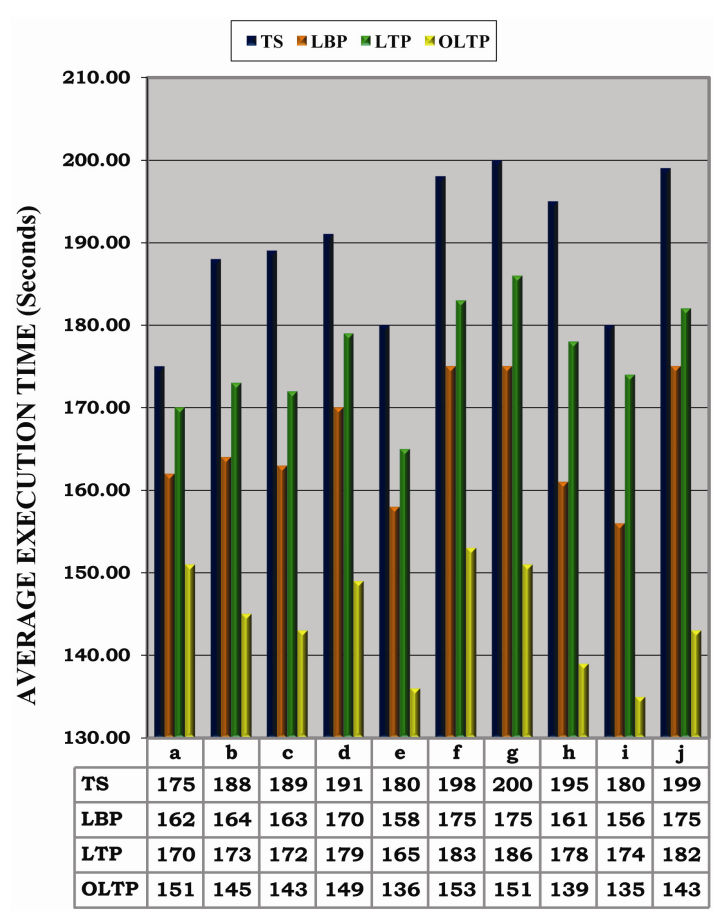

IMAGES(SUBJECTS)

Fig. 8. Computational cost analysis for the face recognition experiments on Yale B face database

and 7 unknown subjects. The system is trained with 40 images of known subjects that are 5 face images from each subject. Testing is done with balance 260 images of both known and unknown subjects. Fig. 7 shows the results of this experiment in the form of a Receiver Operating Characteristic curve (ROC).

Receiver Operating Characteristic (ROC) curves are used for measuring the accuracy of any biometric system at a particular confidence level, in this case it is FAR. The texture model with highest GAR at a particular level of FAR is always considered as the best biometric matcher. From Fig. 7, it can be easily concluded that for all the FAR 
values the corresponding GAR values for the texture model OLTP are relatively higher than all other texture models considered in this comparative study. The result of this comparative study once again proves that OLTP can be a perfect tool for the face recognition also in addition to texture analysis.

In order to evaluate the performance of the selected four texture models for the bench mark of execution time, a subset of 4 different frontal face images from 10 different subjects are randomly selected from the already selected set of frontal face images of Yale B face image database. Fig. 8 illustrates the average computational cost taken by the four texture methods for the selected subjects in face recognition.

As TS model is using very large number of bins in the pattern histogram (6561), it took more execution time for giving the result. In the case of LBP, since this texture model uses 256 patterns it took less time than LTP and TS for giving the output. LTP performs better than TS and OLTP gave the result in the minimum time. It can be observed that OLTP outperforms all other texture models in the process of face recognition because it gave best performance in all assessment factors.

\subsection{Results on ORL face database}

In order to further evaluate the performance of OLTP on face recognition system, another well known face database called ORL database is used in this section. In ORL face database, there are 40 subjects with each subject has 10 different face images. Ten individuals are selected first and then 10 frontal images that is one frontal image from each selected subject are used for training. Fifty frontal images from the remaining images of the same selected subjects which include both gallery images as well as non gallery images are used for testing. Fig. 9 shows some sample images which are selected for this experiment. All the selected images for face recognition are down sampled into $64 \times 64$ size and are converted into gray scale images before experiments. This experiment also compares the face recognition performance of texture descriptors TS, LBP, LTP and OLTP. The results obtained for this experiment is shown in Table 5. Table 5 lists and compares the face recognition results of four texture methods on ORL face database and it is clear that OLTP was the best among all texture models used.

\subsection{Results on AR face database}

In this experiment, this proposed face recognition system which is based on the newly proposed texture model OLTP, uses AR face database for finding the adaptablity of this proposed face recognition system for well recognized AR face database. The AR face database consists of 4,000 frontal images for 135 individuals. For each subject, upto 26 face images are available which are
Table 5. Face Recognition accuracy results on ORL Face Database

\begin{tabular}{c|c|c|c}
\hline $\begin{array}{c}\text { No.of Training } \\
\text { Images }\end{array}$ & $\begin{array}{c}\text { No.of Testing } \\
\text { Images }\end{array}$ & $\begin{array}{c}\text { Texture } \\
\text { Model }\end{array}$ & $\begin{array}{c}\text { Recognition } \\
\text { Rate( } \%)\end{array}$ \\
\hline \multirow{4}{*}{10} & \multirow{3}{*}{50} & TS & 62.23 \\
\cline { 3 - 4 } & & LBP & 78.88 \\
\cline { 3 - 4 } & & LTP & 83.19 \\
\cline { 3 - 4 } & & OLTP & 92.93 \\
\hline
\end{tabular}

Table 6. Face Recognition accuracy results on AR Face Database

\begin{tabular}{c|c|c|c}
\hline $\begin{array}{c}\text { No.of Training } \\
\text { Images }\end{array}$ & $\begin{array}{c}\text { No.of Testing } \\
\text { Images }\end{array}$ & $\begin{array}{c}\text { Texture } \\
\text { Model }\end{array}$ & $\begin{array}{c}\text { Recognition } \\
\text { Rate(\%) }\end{array}$ \\
\hline \multirow{2}{*}{20} & \multirow{3}{*}{100} & TS & 60.07 \\
\cline { 3 - 4 } & & LBP & 82.35 \\
\cline { 3 - 4 } & & LTP & 86.91 \\
\cline { 3 - 4 } & & OLTP & 90.88 \\
\hline
\end{tabular}

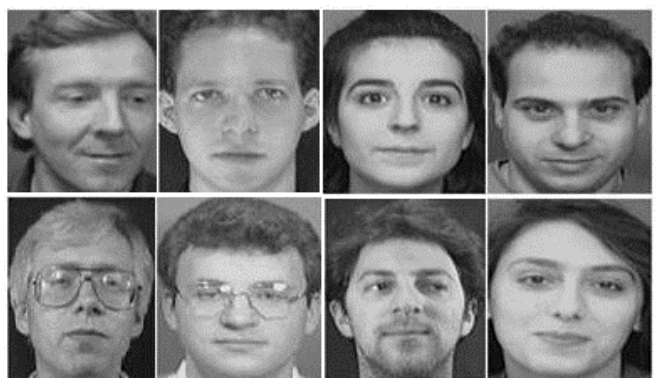

Fig. 9. Sample images-From ORL face database

taken under different variations in illuminations, expressions and occlusions. Twenty individuals (frontal images) are selected first and then 20 frontal images that is one frontal image from each selected subject are used for training. One hundred frontal images from the remaining images of the same selected subjects which include both gallery images as well as non gallery images are used for testing. All the selected images are down sampled into $64 \times 64$ size and are converted into gray scale images before experiments. Fig. 10 shows the sample images selected for this experiment. This experiment only compares the face recognition performance and running time analysis of texture descriptors TS, LBP, LTP and OLTP. The results obtained for this experiment is shown in Table 6 and it is obvious that OLTP was the best among all texture models used.

To study the performance evaluation of various texture models based upon the execution time for face recognition problem on AR database face images, 40 frontal face images are randomly selected from 8 individuals that is in the manner of 5 from each subject. Fig. 11 shows the average computational cost for the selected four texture models for the face recognition over each subject. Obviously as TS model is using large number of bins in the pattern histogram (6561), it took more execution time for giving the result. As expected, in the case of LBP, since this texture model uses 256 patterns it took less time than 

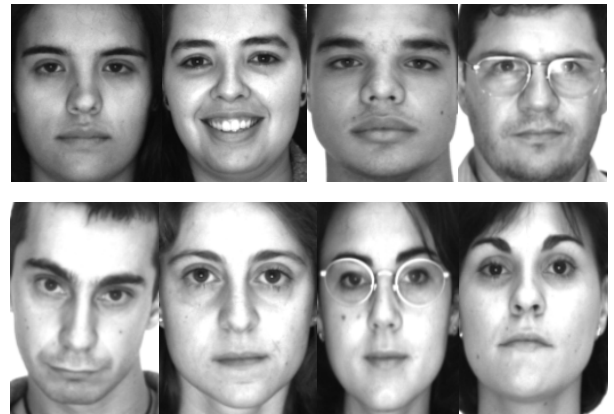

Fig. 10. Sample images-From AR face database

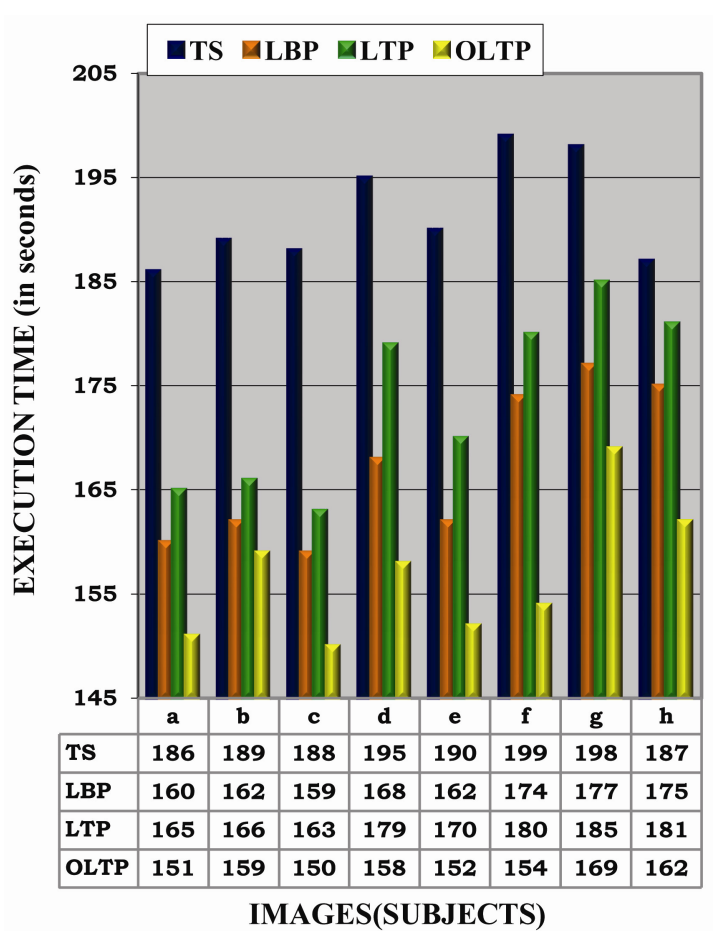

Fig. 11. Computational cost (in seconds) for selected texture models for the face recognition on AR face database

LTP and TS for giving the output. Though LTP gave the result in less time compared to TS, its time complexity is high when compared with OLTP and LBP. Moreover, it is clearly observed that OLTP outperforms all other selected texture models in this experiment of time complexity analysis of face recognition.

\section{Conclusion and Future Scope}

This paper investigates the feasibility and effectiveness of using Optimized Local Ternary Patterns (OLTP) for face representation and face recognition. All the recent state of the art techniques like FAR, FRR, EER and GAR to find the accuracy of the proposed face recognition system have been applied in various experiments. Experimental results on publicly available face databases Yale B, ORL and AR face databases demonstrate that OLTP outperforms other well known texture descriptors namely Texture Spectrum, Local Binary Patterns and Local Ternary Patterns. In future, efforts will be focused on the investigation of the capacity of OLTP for other objects recognition like text detection, person detection, vehicle detection etc. Geometrical features can also be combined with OLTP for future research to achieve better face recognition accuracy. In future, OLTP can be tested for describing face efficiently for expression recognition also. Future work may also include issues that are not considered in this paper like pose variation in the faces, partially occluded face images, face images which are captured under different lighting conditions, noisy face images, etc.

\section{References}

[1] P. Ekman and W. Friesen, "Constants across cultures in the face and emotion," Journal of personality and social psychology, vol. 17, no. 2, pp. 124-129, February 1971.

[2] M. Turk and A. Pentland, "Eigenfaces for recognition," Journal of Cognitive Neuroscience, vol. 3, no. 1, pp. 71- 86, March 1991.

[3] L. Wiskott, J. M. Fellous, N. Kuiger, and C. von der Malsburg, "Face recognition by elastic bunch graph matching," IEEE Transactions on Pattern Analysis and Machine Intelligence, vol. 19, no. 7, pp. 775-779, July 1997.

[4] J. Kittler, Y. Li and J. Matas, "On matching scores for LDA-based face verification," in the proceedings of British Machine Vision conference (BMVC), M. Mirmehdi and B. Thomas Eds., pp. 42-51, September 2000.

[5] F. Cardinaux, C. Sanderson and S. Bengio, "Face verification using adapted generative models," in the proceedings of 6th IEEE International Conference on Automatic Face and Gesture Recognition (AFGR), pp. 825-830, Seoul, May 2004.

[6] E. Hadjidemetriou, M. D. Grossberg and S K. Nayar, "Multiresolution histograms and their use for recognition," IEEE Transactions on Pattern Analysis and Machine Intelligence, vol. 26, no. 7, pp. 831-847, Jul 2004.

[7] P. Yang, S. G. Shan, W. Gao, S. Z. Li and D. Zhang, "Face recognition using Ada-Boosted Gabor features," in the proceedings of 6th IEEE International Conference on Automatic Face and Gesture Recognition (AFGR), pp. 356-361, Seoul, May 2004.

[8] Timo Ahonen, Abdenour Hadid and Matti Pietikainen, "Face Description with Local Binary Patterns: Application to Face Recognition," IEEE Transactions on Pattern Analysis and Machine Intelligence, vol. 28, no. 12, pp. 2037-2041, June 2006.

[9] Shengcai Liao, Xiangxin Zhu, Zhen Lei, Lun Zhang 
and Stan Z. Li., "Learning multi-scale block Local Binary Patterns for Face Recognition," in the Proceedings of International Conference on Biometrics (ICB), Advances in Biometrics, Lecture Notes in Computer Science, vol. 4642, pp. 828-837, Seoul, August 2007.

[10] Amnart Petpon and Sanun Srisuk, "Face Recognition with Local Line Binary Pattern," in the proceedings of fifth IEEE International Conference on Image and Graphics (ICIG), pp. 533-539, China, September 2009.

[11] R. Reena Rose and A. Suruliandi, "Combining Texture with Geometry for performance enhancement of Facial Recognition techniques," in the proceedings of IEEE International Conference on Emerging Trends in Electrical and Computer Technology (ICETECT), pp. 820-825, Tamil Nadu, March 2011.

[12] F. R. Al-Osaimi, Mohammed Bennamoun and Ajmal Mian, "Spatially Optimized Data-level Fusion of Texture and Shape for Face Recognition," IEEE Transactions on Image Processing, vol. 21, no. 2, pp. 859-872, February 2012.

[13] B. Heisele, P. Ho, J. Wa and T. Poggio, "Face recognition: component-based versus global approaches," Computer Vision and Image Understanding, vol. 91, no. 1-2, pp. 6-21, July/August 2003.

[14] Rabin Jafri and Hamid R. Arabina, "A survey of Face Recognition Techniques," Journal of Information Processing Systems, vol. 5, no. 2, pp. 41-68, June 2009.

[15] A. M. Martinez and A. C. Kak, "PCA versus LDA," IEEE Transactions on Pattern Analysis and Machine Intelligence, vol. 23, no. 3, pp. 228-233, February 2001.

[16] H. Shin, S. D. Kim, and H. C. Choi, "Generalized elastic graph matching for face recognition," Pattern Recognition Letters, vol. 28, no. 9, pp. 1077-1082, July 2007.

[17] A. Albiol, D. Monzo, A. Martin, J. Sastre, and A. Alibol, "Face recognition using HOG-EBGM," Pattern recognition Letters, vol. 29, no. 10, pp. 1537-1543, July 2008.

[18] G. Madasamy Raja and V. Sadasivam, "Optimized Local Ternary Patterns: A new Texture Model with set of Optimal Patterns for Texture Analysis," Journal of Computer Science, vol. 9, no. 1, pp. 1-14, Jan 2013.

[19] G. Madasamy Raja and V. Sadasivam, "Segmentation of Multi-Textured images using Optimized Local Ternary Patterns," International Journal of Computer Applications, vol. 95, no. 16, pp. 22-29, June 2014.

[20] L. Wang and DC. He, "Texture Segmentation Using Texture Spectrum," Pattern Recognition, vol. 23, no. 8, pp. 905-910, 1990.

[21] T. Ojala, M. Pietikainen and T. Maenpaa, "Multiresolution gray-scale and rotation invariant texture segmentation with local binary patterns," IEEE Transactions on Pattern Analysis and Machine
Intelligence, vol. 24, no. 7, pp. 971-987, July 2002.

[22] Abhilasha A Patil and Lakshmi Maka, "User Recognition Based on Face using Local Binary Pattern (LBP) with Artificial Neural Network (ANN)," International Journal of Ethics in Engineering \& Management Education, vol. 2, no. 5, pp. 32-35, May 2015.

[23] X. Tan and B. Triggs, "Enhanced local Texture feature sets for Face Recognition under difficult lighting conditions," IEEE Transactions on Image Processing, vol. 19 , no. 6. pp. 1635-1650, June 2010.

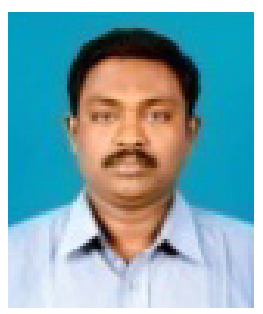

G. Madasamy Raja has obtained his B.E. and M.E., through Manonmaniam Sundaranar University, Tirunelveli, India. He was a Gold medalist in M.E. He has published research papers in many international and national journals. He has also presented research papers in many international and national conferences. His areas of interest in research are Image processing, Fuzzy Logic and Network Security.

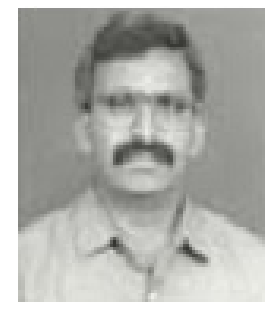

V. Sadasivam has obtained his B.E in Electrical Engineering and M.Sc(Engg) in Power System in 1973 and 1975 respectively. He has completed his $\mathrm{Ph} . \mathrm{D}$ in Computer Science and Engineering in 1993. He is a former Professor and Head of the Department of Computer Science and Engineering at Manonmaniam Sundaranar University, Tirunelveli. His areas of Interest are Software Engineering, Artificial Intelligence, Image Processing and Fuzzy logic. 